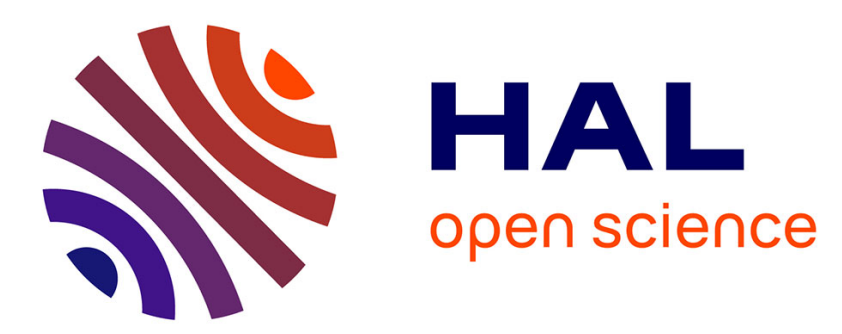

\title{
Theoretical and experimental study of micropolar elastic materials using acoustic waves in air
}

Erick Ogam, Zine El Abiddine Fellah, Mohamed Fellah, Claude Depollier

\section{To cite this version:}

Erick Ogam, Zine El Abiddine Fellah, Mohamed Fellah, Claude Depollier. Theoretical and experimental study of micropolar elastic materials using acoustic waves in air. Journal of Sound and Vibration, 2021, 510, pp.116298. 10.1016/j.jsv.2021.116298 . hal-03266564

\section{HAL Id: hal-03266564 https://hal.science/hal-03266564}

Submitted on 11 Oct 2021

HAL is a multi-disciplinary open access archive for the deposit and dissemination of scientific research documents, whether they are published or not. The documents may come from teaching and research institutions in France or abroad, or from public or private research centers.
L'archive ouverte pluridisciplinaire HAL, est destinée au dépôt et à la diffusion de documents scientifiques de niveau recherche, publiés ou non, émanant des établissements d'enseignement et de recherche français ou étrangers, des laboratoires publics ou privés. 
Erick Ogam ${ }^{\mathrm{a}, *}$, Zine El Abbidine Fellah ${ }^{\mathrm{a}}$, Mohamed Fellah ${ }^{\mathrm{b}}$, Claude Depollier

${ }^{a}$ Laboratoire de Mécanique et d'Acoustique LMA - UMR 7031 AMU - CNRS - Centrale Marseille

${ }^{b}$ Laboratoire de physique théorique, Faculté de physique, Université des Sciences et de la Technologie

$6 \quad$ Houari Boumediene, BP 32 El Alia 16111 Bab Ezzouar Alger, Algeria.

$7 \quad{ }^{c}$ Laboratoire d'Acoustique de l'Université du Mans - UMR CNRS 6613 (LAUM), Avenue Olivier

Messiaen 72085 - Le Mans cedex 9 France.

\section{Abstract}

This study focuses on the development of a theoretical and experimental acoustic method and setup for studying the micropolar behavior of materials. Two types of low density closed-cell foams made from polystyrene (expanded and extruded) materials that were previously reported as materials exhibiting the non-classical elastodynamic behavior were investigated in order to reveal the particular comportment using acoustic waves. Two different types of transducers were employed to provide a large frequency band to study the influence of the Cosserat parameters. The frequency band from $1-40 \mathrm{kHz}$ was covered using a twitter loudspeaker whereas that from $30-120 \mathrm{kHz}$, by an air coupled ultrasonic transducer. The materials' micropolar elastic behavior were revealed through the comparison between the theoretical and experimental transmission coefficients. At the cut-off frequency where the theoretical phase velocity of the transverse acoustic mode was established to be infinite, the acoustic wave transmission by the panels was found to be very small. This makes the Cosserat materials appealing to harmonic noise reduction.

*Principal corresponding author

Email address: ogam@lma.cnrs-mrs.fr (Erick Ogam) 
13 The Cosserat brothers, Eugène and François, published a monograph [1] in 1909 in 14 which they presented a new variant of Continuum Mechanics. This detailed treatise 15 covered the idea of taking into account additionally the couple stresses, the rotational 16 degrees of freedom (micro-rotations) and their gradients of the material particles as 17 independent variables. The existence of the couple stresses was originally postulated by 18 Voigt in 1887 [2].

The couple stress theory using macrorotation as the true kinematical rotation for elastic bodies in which the gradient of the rotation vector is used as a curvature tensor was developed much later by Toupin [3, Mindlin and Tiersten [4, Koiter [5]. These formulations use the four foundational continuum mechanical quantities (i.e., force, displacement, couple, rotation) and are the fundamental pillars in the development of size-dependent continuum mechanics. Heterogeneous materials exhibit intrinsic material length scales (like in cellular structures, grains, particles and fibers). However, these formulations pose some challenges like the indeterminacy of the spherical part of the couple-stress tensor and the appearance of the body couple in the constitutive relation for the force-stress tensor [4, 6]. Hadjesfandiari and Dargush [7] resolved the inconsistencies by revealing

29 the subtle skew-symmetric character of the couple-stress tensor and showed that it was

sо a true vector. The constrained Cosserat theory is a particular case of the couple stress

31 theory in which kinematic constraints have been introduced in order to eliminate some

32 of the degrees of freedom [8] in order to obtain a simpler system of equations.

33 The extension of the linear Cosserat theory to include body microinertia effects is ${ }_{34}$ attributed to Eringen[9]. He is also the one who renamed it as the micropolar theory

35 of elasticity [10. A complete variant of this theory can be found in Kafadar and Erin-

36 gen [11. Micropolar continuum mechanics [12] therefore incorporates a local rotation of

37 points (micropolar continua in the sense of Eringen) including the translation assumed

38 in classical elasticity; and a couple stress as well as the force stress.

39 Six material parameters are needed in the linear micropolar elasticity of isotropic 
40 solids while only two Lamé moduli are necessary in the classical elasticity. A review and

41 survey of the literature on the theory of Cosserat continuum can be found in [13, 14].

${ }_{42}$ This theory has been extended further to capture more complex micropolar material

43 behavior [15-17.

44 The majority of studies pertaining to the micropolar elastodynamics of materials

45 have been mainly theoretical. The most popular media geometry studied was that of an

46 infinite half-plane corresponding to the ground in geology and seismic studies. Many of

47 the problems reported were those concerning reflection and refraction of plane waves at

48 plane interfaces in which seismic waves generated within a semi-infinite half-plane mi-

49 cropolar solid media interact with, for example, a viscous liquid layer [18, 19]. The few

so experimental measurements reported were mainly undertaken using mechanical (static,

51 quasi-static) means [20-23]. All six elastic constant parameters (like the apparent shear

52 modulus) have been found from torque and twist measurements in a pure torsion exper-

53 iment using a circular cylinder and the cylindrical bending of a rectangular plate [24].

$54 \quad$ In this study, a theoretical micropolar elastic model was developed and employed to

55 validate a new experimental method to study acoustic wave transmission through air-

56 filled closed-cell polystyrene panels considered to obey Cosserat elasticity (micropolar

57 continuum theory) law. The frequency domain ranges from the audible to the ultrasonic

58 regimes but $<120 \mathrm{kHz}$. The foams studied were two-phase composite materials in which

59 one phase was solid and the other, fluid (air). In this case, the size scale is large, the

60 material may no longer be assumed to be continuous [25, 26. A table in reference [14]

${ }_{61}$ classified the closed-cell polystyrene foams in the family of Cosserat elasticity materials.

62 The micropolar continuum theory model considered herein was that of the generalized

${ }_{63}$ plane strain conditions assuming that the strain distributions do not depend on the third

64 coordinate geometry of the acoustic wave transmission through panels experiment. The

65 other reason motivating this study is that elastic data for closed-cell materials like the

66 EPS and XPS foams are hard to come by in the literature and the only existing methods

67 used by manufacturers are based on the static destructive mechanical methods declined 
68 as standard test methods [27, 28].

\section{2. Theoretical and experimental methods}

7o 2.1. Theoretical method for wave propagation in a Cosserat continuum

71 The loadings on a micropolar continuum of density $\rho$ and rotational inertia $J$ generate 72 deformation of the body described by the displacement vector $\mathbf{u}$ and the rotation vector $73 \Phi$.

74 The equations of motion in the micropolar elastic continuum are [20, 29]

$$
\begin{aligned}
& (\lambda+2 \mu+\mathcal{K}) \nabla(\nabla \bullet \mathbf{u})-(\mu+\mathcal{K}) \nabla \times(\nabla \times \mathbf{u})+\mathcal{K} \nabla \times \boldsymbol{\Phi}-\rho \frac{\mathrm{d}^{2}}{\mathrm{~d} t^{2}} \mathbf{u}=0, \\
& (\alpha+\beta+\gamma) \nabla(\nabla \bullet \boldsymbol{\Phi})-\gamma \nabla \times \nabla \times \boldsymbol{\Phi}+\mathcal{K} \nabla \times \mathbf{u}-2 \mathcal{K} \boldsymbol{\Phi}-\rho J \frac{\mathrm{d}^{2}}{\mathrm{~d} t^{2}} \boldsymbol{\Phi}=0,
\end{aligned}
$$

${ }_{75} \quad$ where $\lambda, \mu$ are the Lamé constants, $\alpha, \beta, \mathcal{K}$ and $\gamma$ are the new elastic constants 76 usually referred to as the micropolar or Cosserat elastic constants.

77 In order to uncouple the equations of motion in Eqn. (1), the Helmholtz decomposi78 tion is employed i.e., decompose the vectors $\boldsymbol{u}$ and $\boldsymbol{\Phi}$ into their potential and solenoidal 79 parts

$$
\begin{aligned}
& \boldsymbol{u}=\nabla q+\nabla \times \boldsymbol{\Pi}, \quad \nabla \bullet \boldsymbol{\Pi}=0, \\
& \boldsymbol{\Phi}=\nabla \xi+\nabla \times \boldsymbol{\Theta}, \quad \nabla \bullet \Theta=0,
\end{aligned}
$$

so The following uncoupled wave equations for the potentials $q$ and $\xi$ are obtained

$$
\begin{aligned}
c_{1}^{2} \nabla^{2} q-\frac{\partial^{2}}{\partial t^{2}} q & =0 \\
c_{3}^{2} \nabla \xi-\omega_{0}^{2} \xi-\frac{\partial^{2}}{\partial t^{2}} \xi & =0
\end{aligned}
$$


81 $\quad$ where $c_{1}^{2}=\frac{\lambda+2 \mu+\mathcal{K}}{\rho}=\frac{M+\mathcal{K}}{\rho}, c_{3}^{2}=\frac{\alpha+\beta+\gamma}{\rho J}, \omega_{0}^{2}=\frac{2 \mathcal{K}}{\rho J}$.

82 The coupled system of wave propagation equations for the determination of the vector

83 potential for $\boldsymbol{\Pi}$ and $\boldsymbol{\Theta}$

$$
\begin{aligned}
c_{2}^{2} \nabla^{2} \boldsymbol{\Pi}+\frac{J \omega_{0}^{2}}{2} \nabla \times \boldsymbol{\Theta}-\frac{\boldsymbol{\partial}^{\mathbf{2}}}{\partial \boldsymbol{t}^{\mathbf{2}}} \boldsymbol{\Pi}=0, \\
c_{4}^{2} \nabla^{2} \boldsymbol{\Theta}-\omega_{0}^{2} \Theta+\frac{\omega_{0}^{2}}{2} \nabla \times \boldsymbol{\Pi}-\frac{\partial^{2}}{\partial t^{2}} \boldsymbol{\Theta}=0 .
\end{aligned}
$$

$84 \quad$ where $c_{2}^{2}=\frac{\mu+\mathcal{K}}{\rho}, c_{4}^{2}=\frac{\gamma}{\rho J}$,

85 These equations govern the propagation of waves travelling in a Cosserat continuum.

s6 The details of deriving the dispersion relations are found in the Appendix A or in 87 30.

88 Finally, there are four different waves propagating with four different phase velocities

$$
v_{1}^{2}=c_{1}^{2}=\frac{\lambda+2 \mu+\mathcal{K}}{\rho}, \quad v_{2}^{2}=c_{3}^{2}+\frac{\omega_{0}^{2}}{k^{2}}, \quad v_{3,4}^{2}=\frac{\omega^{2}}{k_{3,4}^{2}}
$$

89 Therefore, in a micropolar elastic medium of infinite extent propagates four types of 9o modes, 1) A longitudinal displacement wave mode propagating with a phase velocity,

$91 \quad v_{1}$ also called Longitudinal Acoustic mode (LA-mode), 2) A longitudinal microrotation 92 wave (L0-mode) propagates with the velocity $v_{2}, 3$ ) Transverse acoustic mode (TA-mode),

${ }_{93}$ propagating with phase velocity $\left.v_{3}, 4\right)$ Transverse Optic mode (TO-mode) propagating

94 with phase velocity $v_{4}$.

95 It can be shown that the velocity of the longitudinal microrotation wave $\left(v_{2}\right)$ is

96 dispersive (depends on frequency) when $\omega>\sqrt{2} \omega_{0}$

$$
v_{2}^{2}=\frac{\alpha+\beta+\gamma}{J \rho\left(1-\frac{2 \omega_{0}^{2}}{\omega^{2}}\right)}
$$

97 However, when $\omega=\omega_{c}=\sqrt{2} \omega_{0}, v_{2}$ is infinite and the wave number equals zero.

98 When $\omega<\omega_{c}, v_{2}$ is purely imaginary. The characteristic frequency $\omega_{c}$ acts as a 
99 cut-off frequency below which the wave number vanishes.

${ }_{100}$ The TO velocity $v_{3}=\infty$ at $\omega=\omega_{c}$. The sketches of the phase velocities $v_{3}$ and $v_{4}$ 101 are given in reference [29]. It was shown that at $\omega=\omega_{c}, v_{3}=\infty$. For $\omega<\omega_{c}^{-}, v_{3}=-\infty$ 102 and at $\omega>\omega_{c}^{+}, v_{3}=+\infty$. The transverse optic mode wave velocity $v_{4}$ is finite over the 103 whole frequency range.

${ }_{104}$ The experimental analysis of the phenomenon occuring around the cut-off frequency

105 of $v_{3}$ was therefore of significant interest in this study.

$$
\boldsymbol{u}=\left(0, u_{y}, u_{z}\right), \quad \boldsymbol{\Phi}=\left(\Phi_{x}, 0,0\right), \quad \partial / \partial x=0
$$

115 An incident acoustic plane wave propagating in the fluid $\Omega_{0}$ impinges on the panel 116 at an incident angle $\theta$ and is reflected at the $z=0$ plane at the boundary $\Gamma_{1}$. Mode 117 transformation takes place i.e, from longitudinal wave in the fluid into the four micropolar 118 elastic wave modes (LA, TA, LO and TO wave modes) in the panel. Multiple internal 119 reflections of one of the modes within the layer is also represented in Fig. (1) for only a 120 single mode for clarity. 


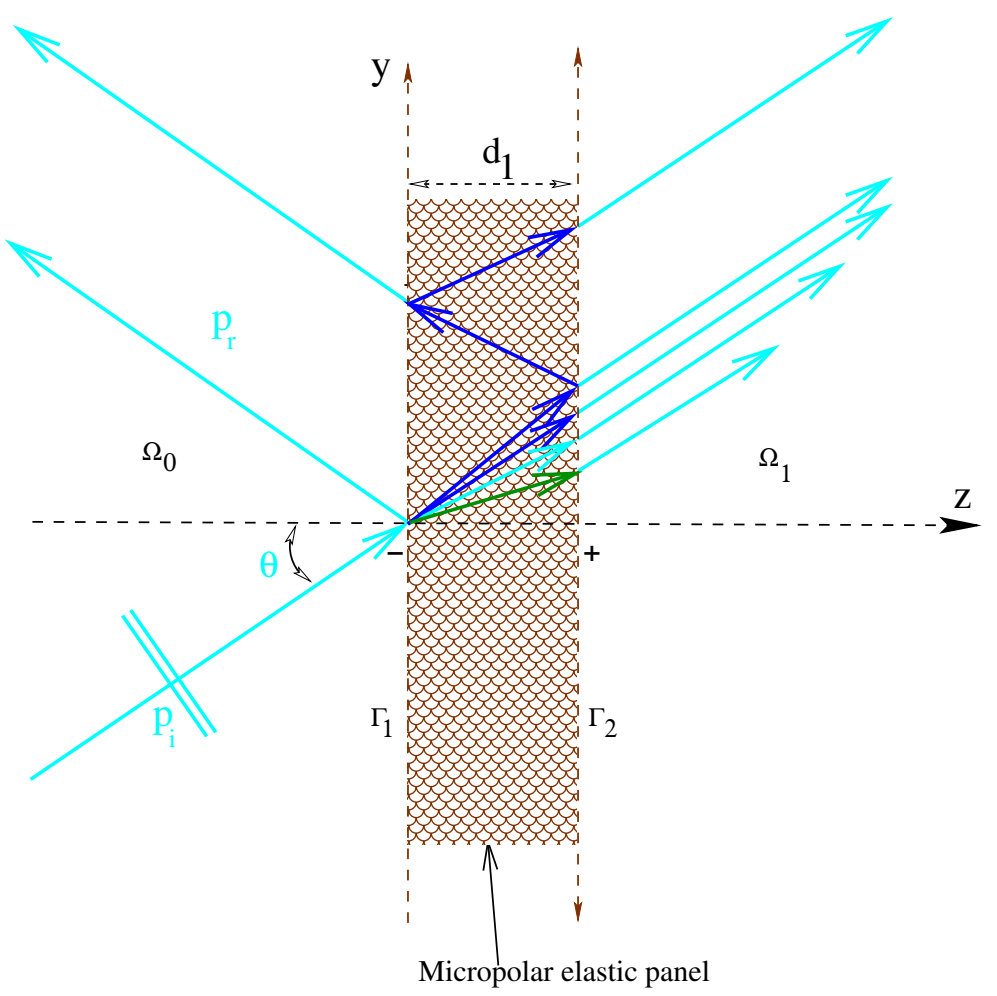

Figure 1: Geometry of the acoustic wave transmission problem. The panel is supposed to extend to infinity in the positive and negative $y$ - directions. 
122 The boundary surface $\Gamma_{1}$ and $\Gamma_{2}$ are free from traction and couples, the equations for 123 the boundary conditions for transmission/reflection through an infinite extent micropolar 124 plate immersed in air. The stress tensors $\sigma_{z z}, \sigma_{y z}$ and the couple stress tensor $m_{y z}$ are 125 employed

$$
\begin{aligned}
\sigma_{z z}(y, z)= & \lambda\left(\frac{\partial^{2}}{\partial y^{2}} q(y, z)+\frac{\partial^{2}}{\partial z^{2}} q(y, z)\right)+(2 \mu+\mathcal{K})\left(\frac{\partial^{2}}{\partial z^{2}} q(y, z)-\frac{\partial^{2}}{\partial z \partial y} \Pi_{x}(y, z)\right) \\
\sigma_{z y}(y, z)= & (2 \mu+\mathcal{K}) \frac{\partial^{2}}{\partial z \partial y} q(y, z)+(\mu+\mathcal{K}) \frac{\partial^{2}}{\partial z^{2}} \Pi_{x}(y, z)-\mu \frac{\partial^{2}}{\partial y^{2}} \Pi_{x}(y, z)+ \\
& \mathcal{K}\left(\frac{\partial}{\partial y} \Theta_{z}(y, z)-\frac{\partial}{\partial z} \Theta_{y}(y, z)\right) \\
m_{z x}(y, z)= & \gamma\left(\frac{\partial^{2}}{\partial z \partial y} \Theta_{z}(y, z)-\frac{\partial^{2}}{\partial z^{2}} \Theta_{y}(y, z)\right) .
\end{aligned}
$$

126 The continuity of the acoustic particle velocity at the boundaries of the panel $\Gamma_{1}$, and ${ }_{127} \Gamma_{2}$, are also sought. The velocity in the panel is given by

$$
v_{z}(y, z)=\mathrm{i} \omega \frac{\partial}{\partial z} q(y, z)-\mathrm{i} \omega \frac{\partial}{\partial y} \Pi_{x}(y, z) \quad z \geq \Gamma_{1}, \cup z \leq \Gamma_{2},
$$

${ }_{128}$ The 8 boundary conditions' equations are set using the following stress, torque and 129 velocity equations

$$
\begin{aligned}
\sigma_{z z}(y, 0) & =-p_{I}, \quad z \in \Gamma_{1}, \sigma_{z z}\left(y, d_{1}\right)=-p_{T} z \in \Gamma_{2}, \\
\sigma_{z y}(y, 0) & =0, \quad z \in \Gamma_{1}, \sigma_{z y}\left(y, d_{1}\right)=0 \quad z \in \Gamma_{2}, \\
m_{y z}(y, z) & =0, \quad z \in \Gamma_{1} \cup \Gamma_{2}, \\
v_{z}^{\Gamma_{1+}}(y, 0) & =v_{z}^{\Gamma_{1-}}(y, 0), \quad v_{z}^{\Gamma_{2+}}\left(y, d_{1}\right)=v_{z}^{\Gamma_{2-}}\left(y, d_{1}\right) .
\end{aligned}
$$

130 The acoustic pressure in the fluid medium at $z \leq \Gamma_{1}\left(p_{I}\right)$ and at $z \geq \Gamma_{2},\left(p_{T}\right)$ are 
131 given by

$$
\begin{aligned}
& p_{I}(y, z)=P_{I}\left(\mathrm{e}^{\kappa_{z}^{f} z}+R \mathrm{e}^{-\kappa_{z}^{f} z}\right) \mathrm{e}^{\kappa_{y}^{f} y}, z \leq \Gamma_{1}, \\
& p_{T}(y, z)=T P_{I} \mathrm{e}^{\left(\kappa_{z 1}+\kappa_{z 3}+\kappa_{z 4}\right) d_{1}+\kappa_{z}^{f}\left(z-d_{1}\right)} \mathrm{e}^{\kappa_{y}^{f} y}, z \geq \Gamma_{2},
\end{aligned}
$$

132 where $R$ and $T$ are the reflection and transmission coefficients respectively, $\kappa_{z}^{f}=$ $133 \frac{\mathrm{i} \omega}{c_{f}} \cos \left(\theta_{I}\right)\left(\theta_{I}\right.$ is the oblique angle of incidence), $\kappa_{y}^{f}=\frac{\mathrm{i} \omega}{c_{f}} \sin \left(\theta_{I}\right), c_{f}$ is the acoustic wave ${ }_{134}$ velocity in the fluid. The wave velocities are computed using the expressions of the fluid 135 pressure above and the linear Euler equation, $v_{z}(y, z)=\frac{-1}{\mathrm{i} \omega \rho_{f}} \frac{\partial}{\partial z} p(y, z)$. The complex 136 wave numbers $\kappa_{z n}=\mathrm{i} k_{n} \cos \left(\theta_{n}\right)(\mathrm{n}=1 \ldots 4)$ for the waves propagating in the micropolar 137 elastic panel.

${ }_{138} \quad$ The incident and reflected waves are indicated by superscripts $I$ and $R$ propagate in 139 the slab. The potentials for the micropolar elastic medium are given by

$$
q=a_{1}^{I} \exp \left[\mathrm{i} k_{1}\left(\sin \theta_{1}^{I} y+\cos \theta_{1}^{I} z\right)-i \omega_{1}^{I} t\right]+a_{1}^{R} \exp \left[\mathrm{i} k_{1}\left(\sin \theta_{1}^{R} y-\cos \theta_{1}^{R} z\right)-\mathrm{i} \omega_{1}^{R} t\right]
$$

$\Pi_{x}=A_{3 x}^{I} \exp \left[\mathrm{i} k_{3}\left(\sin \theta_{3}^{I} y+\cos \theta_{3}^{I} z\right)-\mathrm{i} \omega_{3}^{I} t\right]+A_{3 x}^{R} \exp \left[\mathrm{i} k_{3}\left(\sin \theta_{3}^{R} y-\cos \theta_{3}^{R} z\right)-\mathrm{i} \omega_{3}^{R} t\right]+$ $A_{4 x}^{I} \exp \left[\mathrm{i} k_{4}\left(\sin \theta_{4}^{I} y+\cos \theta_{4}^{I} z\right)-\mathrm{i} \omega_{4}^{I} t\right]+A_{4 x}^{R} \exp \left[\mathrm{i} k_{4}\left(\sin \theta_{4}^{R} y-\cos \theta_{4}^{R} z\right)-\mathrm{i} \omega_{4}^{R} t\right]$,

Since the transverse TA and TO waves are coupled we can write

$$
\begin{aligned}
& \Theta_{y}=\eta_{3 y}\left\{A_{3 x}^{I} \exp \left[\mathrm{i} k_{3}\left(\sin \theta_{3}^{I} y+\cos \theta_{3}^{I} z\right)-\mathrm{i} \omega_{3}^{I} t\right]+A_{3 x}^{R} \exp \left[\mathrm{i} k_{3}\left(\sin \theta_{3}^{R} y-\cos \theta_{3}^{R} z\right)-\mathrm{i} \omega_{3}^{R} t\right]\right\}+ \\
& \eta_{4 y}\left\{A_{4 x}^{I} \exp \left[\mathrm{i} k_{4}\left(\sin \theta_{4}^{I} y+\cos \theta_{4}^{I} z\right)-\mathrm{i} \omega_{4}^{I} t\right]+A_{4 x}^{R} \exp \left[\mathrm{i} k_{4}\left(\sin \theta_{4}^{R} y-\cos \theta_{4}^{R} z\right)-\mathrm{i} \omega_{4}^{R} t\right]\right\}
\end{aligned}
$$


$\Theta_{z}=\eta_{3 z}\left\{A_{3 x}^{I} \exp \left[\mathrm{i} k_{3}\left(\sin \theta_{3}^{I} y+\cos \theta_{3}^{I} z\right)-\mathrm{i} \omega_{3}^{I} t\right]+A_{3 x}^{R} \exp \left[\mathrm{i} k_{3}\left(\sin \theta_{3}^{R} y-\cos \theta_{3}^{R} z\right)-\mathrm{i} \omega_{3}^{R} t\right]\right\}+$ $\eta_{4 z}\left\{A_{4 x}^{I} \exp \left[\mathrm{i} k_{4}\left(\sin \theta_{4}^{I} y+\cos \theta_{4}^{I} z\right)-\mathrm{i} \omega_{4}^{I} t\right]+A_{4 x} \exp \left[\mathrm{i} k_{4}\left(\sin \theta_{4}^{R} y-\cos \theta_{4}^{R} z\right)-\mathrm{i} \omega_{4}^{R} t\right]\right\}$,

140 where the coupling coefficients $\eta_{3,4}$ were derived from Eqn. A.3. using the amplitude

141 ratios

$$
\overline{\boldsymbol{\Theta}}_{y}=\frac{-\mathrm{i} \omega_{0}^{2}}{2\left(\omega^{2}-\omega_{0}^{2}-c_{4}^{2} k^{2}\right)} k_{z} \bar{\Pi}_{x}, \quad \overline{\boldsymbol{\Theta}}_{z}=\frac{\mathrm{i} \omega_{0}^{2}}{2\left(\omega^{2}-\omega_{0}^{2}-c_{4}^{2} k^{2}\right)} k_{y} \bar{\Pi}_{x}
$$

142 and finally the amplitude ratios

$$
\eta_{3,4 y}=-\frac{\mathrm{i} \omega_{0}^{2}}{2 k_{3,4}\left(v_{3,4}^{2}-\frac{\omega_{0}^{2}}{k_{3,4}^{2}}-c_{4}^{2}\right)} \cos \theta_{3,4}, \quad \eta_{3,4 z}=\frac{\mathrm{i} \omega_{0}^{2}}{2 k_{3,4}\left(v_{3,4}^{2}-\frac{\omega_{0}^{2}}{k_{3,4}^{2}}-c_{4}^{2}\right)} \sin \theta_{3,4}
$$

143 The complex wave-numbers $\kappa_{f}=\mathrm{i} \vartheta_{f}, \vartheta_{f}=k_{f} \sin \left(\theta_{I}\right)\left(k_{f}\right.$ is the compressional wave ${ }_{144}$ number in the fluid). Simplifications can be done on the potentials by writing $\theta_{n}^{I}=$ ${ }_{145} \theta_{n}^{R}(n=1 \ldots 4)$. The complex wave numbers $\kappa_{z n}=\mathrm{i} k_{n} \cos \left(\theta_{n}\right)$ for the four waves ${ }_{146}$ propagating in the micropolar elastic panel are related to the incident wave number in 147 the fluid through Snell-Descartes law of refraction and therefore can be written

$$
\begin{aligned}
& \kappa_{z 1}=\mathrm{i} \sqrt{k_{1}^{2}-\vartheta_{f}^{2}}, \quad \kappa_{z 2}=\mathrm{i} \sqrt{k_{2}^{2}-\vartheta_{f}^{2}} \\
& \kappa_{z 3}=\mathrm{i} \sqrt{k_{3}^{2}-\vartheta_{f}^{2}}, \quad \kappa_{z 4}=\mathrm{i} \sqrt{k_{4}^{2}-\vartheta_{f}^{2}}
\end{aligned}
$$

${ }_{148}$ The hysteretic model of inherent damping of the panels were taken into account by 149 introducing a scalar-valued loss factor $\chi(\omega)$ into Young's modulus 31, such that $E_{d}(\omega)=$ $150 \quad E_{r}(\omega)+\mathrm{i} E_{i}(\omega)=E(\omega)(1+\mathrm{i} \chi(\omega))\left(E_{r}(\omega)\right.$ is called the storage modulus and $E_{i}(\omega)$ the 151 loss modulus of the panel, $M(\omega)=\frac{E(\omega)(1-\nu)}{(1+\nu)(1-2 \nu)}$ where $\nu$ is the Poisson ratio). $E(\omega)$ 152 is approximately constant in the chosen bandwidth (there is a basic lag between the 153 stress and the strain and therefore the area of the hysteresis cycle does not depend on 154 the frequency of the harmonic motion). The relationships between the different elastic 
155 moduli can be found in reference 32 .

156 A matrix equation is deduced from the 8 boundary conditions' equations

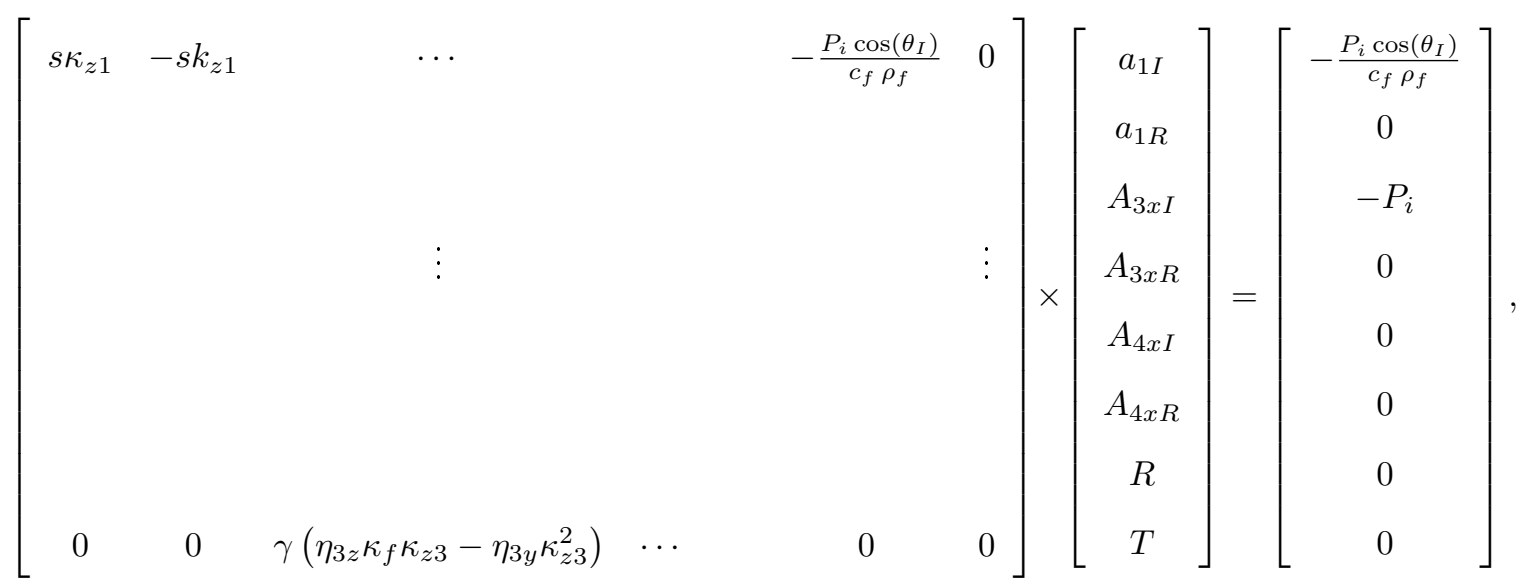

157 where $s=i \omega$.

158 The matrix equation is written as a linear system in the form $\boldsymbol{A} \boldsymbol{x}=\boldsymbol{b}(\boldsymbol{A}$ is an $8 \times 8$

159 matrix) and the equation solved to obtain $T$ or $R$.

165 made from extruded sheets 33 .

166 The experimental setup is shown in Fig. 2. A transient pulse wave was generated 167 using a loudspeaker driven by a waveform function generator (Agilent 33250B, Loveland 168 Colorado, USA) through a power amplifier Bruel and Kjaer (B\&K2706, Naerum Den169 mark). The excitation signal generated was a positive rectangular pulse. The Fourier 170 transform of the excitation pulse $g(t)$ with amplitude $A$ and width $P_{w d t}$ in seconds is

$$
G(f)=A P_{w d t} \operatorname{sinc}\left(\pi f P_{w d t}\right) .
$$


${ }_{171}$ As $P_{w d t}$ becomes very large, the magnitude spectrum approaches a Dirac delta function 172 located at the origin. As the height of the pulse becomes higher and its width smaller, it ${ }_{173}$ approaches a Dirac delta function and the magnitude spectrum flattens out and becomes ${ }_{174}$ a constant. This latter case results in a signal with large frequency band-width being 175 generated. Shorter pulse widths also avoid the risk of damaging the loud-speaker when 176 the amplitude is high. When the panel is thin, the first mode has a high frequency 177 value, therefore, $P_{w d t}$ is smaller, and relatively wider for a thick panel. The pulse width ${ }_{178}$ was chosen $5 \mu \mathrm{s}<P_{w d t}<12 \mu \mathrm{s}$. Even though the generated pulse is rectangular form, 179 the captured pulse of the incident wave will look sinusoidal because the generator signal 180 output is convoluted with the response of the loud-speaker.

181 The chosen loudspeaker was a Visaton (GmbH, Haan, Germany) magnetostatic rib182 bon tweeter reference MHT 12 - 8 Ohm with an opening, $60 \mathrm{~mm}$ wide and $\times 84 \mathrm{~mm}$ ${ }_{183}$ high. It was selected among several others because it had a low distortion factor, good 184 power-handling capabilities (100 Watts), highly linear impedance and amplitude fre185 quency response and a broad frequency response range $(1 \mathrm{kHz}-50 \mathrm{kHz})$. This is the 186 first time such a tweeter speaker is being employed in this frequency range. This wide 187 range is an advantage over the non-contact air-coupled ultrasonic transducers that are ${ }_{188}$ band limited around their central working frequency e.g the Ultran NCG50-S50 (State 189 College, PA USA) is a $50 \mathrm{kHz}$ center frequency transducer whose band-with is $\approx 20 \mathrm{kHz}$. 190 The transmitted acoustic wave was captured using a small ( $1 / 8$ inch) pressure-field 191 condenser microphone (Type B\&K4138, Naerum Denmark) connected to a frequency 192 analyzer (B\&K2120, Naerum Denmark). The very low noise and high amplification an193 alyzer was used in the measuring microphone amplifier mode $(2 \mathrm{~Hz}-200 \mathrm{kHz})$. The 194 signals from the microphone amplifier were then digitized using a Tektronix oscilloscope 195 model TDS3014B (Beaverton, OR USA). The transmission coefficient was obtained by 196 calculating the transfer function estimate between the captured incident acoustic pres197 sure(obtained without the panel in place). The transmitted acoustic pressure was ac198 quired when the panel was placed perpendicularly between the acoustic source and the 
sensor (microphone).

The transmission coefficient was computed as a transfer function of a linear time-

201 invariant (LTI) system giving the ratio between the incident pressure and the transmitted

202 acoustic pressure. The transmission coefficient $T(f)$ was obtained from the quotient of

203 the cross spectral power density $S_{i t}$ (between the incident, $p_{i}(t)$ and the transmitted,

$\left.{ }_{204} p_{t}(t)\right)$ acoustic pressure) and the auto-spectral power density $S_{i i}\left(p_{i}(t)\right)$,

$$
\tilde{T}(f)=\frac{\tilde{S}_{i t}(f)}{\tilde{S}_{i i}(f)} .
$$

205 where the tilde $(\sim)$ indicates that the function is an estimate only. The quotient $\tilde{T}(f)$

206 was calculated in Matlabß employing the function tfestimate 34 using the acquired

207 signals. In order to avoid spectral leakage and attain accurate spectral measurements, the temporal signals were first windowed [35].

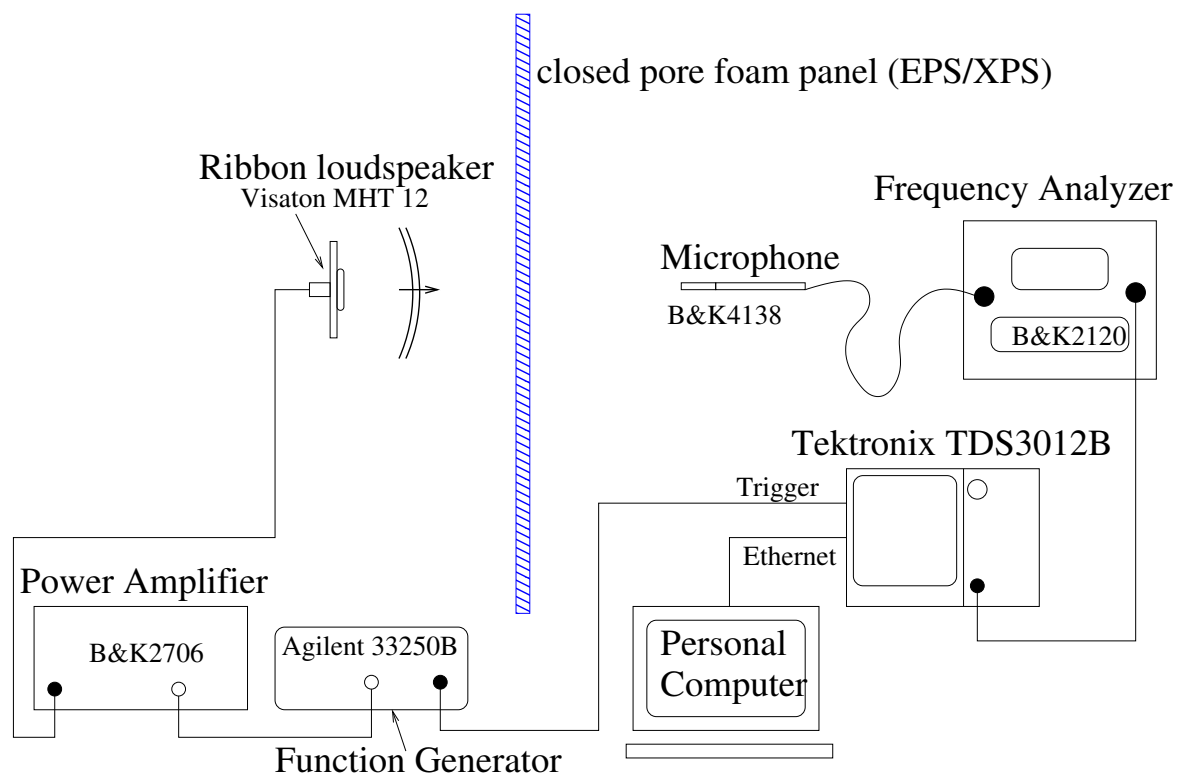

Figure 2: The experimental setup for the acoustic wave transmission by a panel. 
234 solid panel layer. width

\section{Results}

\subsection{XPS Panels} signal processing.

Using a $50 \mathrm{kHz}$ ultrasonic transducer as acoustic source to extend the measurement band-

The measurement frequency bandwidth beyond $40 \mathrm{kHz}$ was extended by replacing the loudspeaker by an air-coupled ultrasonic probe as an acoustic excitation source [36].

The exciter probe (Ultran NCG50-S50, State College, PA USA) had a center frequency at $50 \mathrm{kHz}$ and was connected to a Panametrics 5058-PR (Waltham USA) pulser. The replacement of the acoustic exciter just modified the experimental setup slightly as the acoustic receiver remained the same as in the loudspeaker source/microphone configuration. If the ultrasonic detector had been used as receiver in place of the microphone, it would have engendered undesirable reflections from its non-negligible larger surface area rendering the modeling more complex. It was actually found to reflect the transmitted waves backwards towards the panels due to its large surface area. The NCG50-S50 has a large $(50 \mathrm{~mm} \times 50 \mathrm{~mm})$ active area. This is the reason why a tiny $1 / 8$ inch in diameter B\&K 4138 microphone was preferred as receiver/detector.

The temporal signals representing the incident pressure (without the $4 \mathrm{~cm}$ thick XPS panel) and transmitted pressure (with the panel placed between the speaker and the microphone) and their frequency spectrum are depicted in Figs. (3) a and b, respectively.

A rectangular window providing a better spectral accuracy was employed during the

From the zoomed view of the temporal signals (Fig. (3)a), it can be noticed that the transmitted acoustic pressure wave is attenuated but arrives at the microphone position earlier than the incident waves. This is because the acoustic waves travel faster in the micropolar elastic solid than in air and are therefore accelerated in a small portion of the

$$
\text { solid panel layer. }
$$



${ }_{236}$ mitted pressure wave is cut-off at around $5-6 \mathrm{kHz}$ (calculated $\omega_{c}$ is $5319 \mathrm{~Hz}$ such that ${ }_{237}$ the amplitude is diminished around this frequency value. The comparison between the ${ }_{238}$ theoretical micropolar elastic model and the experimentally determined transmission co${ }_{239}$ efficients (TC) are shown in Fig. (3)c. The theoretical cut-off frequency $f_{c}$ is $\approx 5319$ $240 \mathrm{~Hz}$.

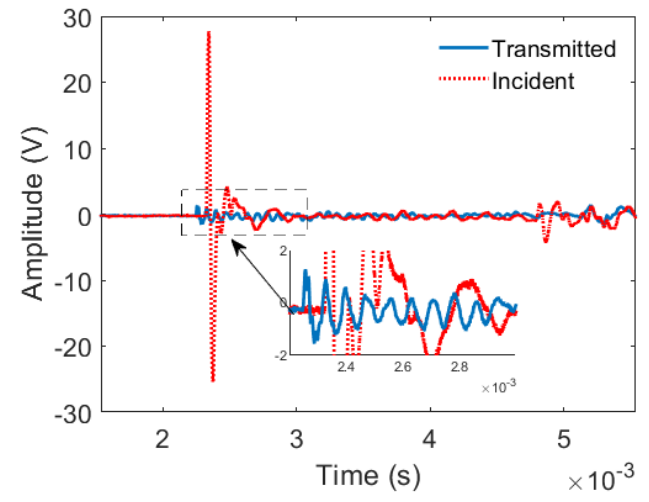

(a)

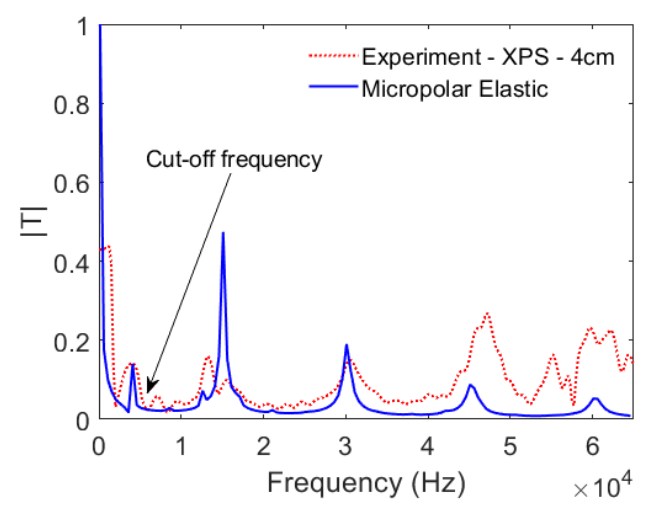

(c)

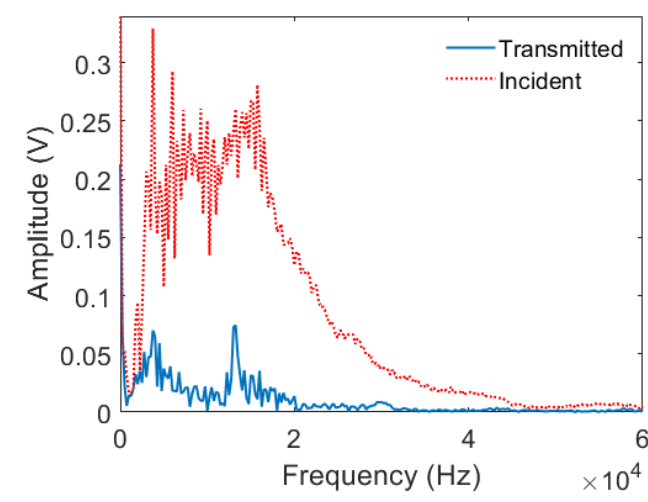

(b)

Figure 3: For the $4.0 \mathrm{~cm}$ thick XPS panel, (a) The temporal signals representing the incident and transmitted pressure. The transmitted wave is attenuated, albeit faster than the incident wave. (b) The frequency spectrum. Comparisons between the theoretical micropolar elastic and classical elastic models with experimental data (c) transmission coefficients. The computed cut-off frequency is at $5319 \mathrm{~Hz}$.

The measurements results for the $2.0 \mathrm{~cm}$ thick XPS panel are shown in Fig. (4) $\mathrm{a}$ - 
242 c. It can be noted that the frequency of the modes also depend on the thickness of the

243 panel. The thicker the panel, the lower the frequency values of the modes.

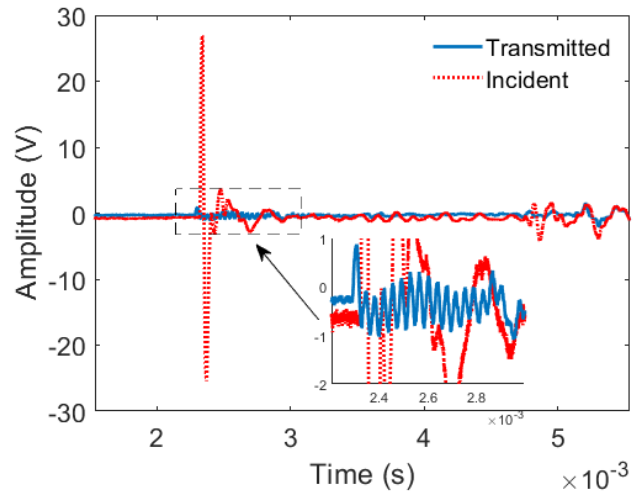

(a)

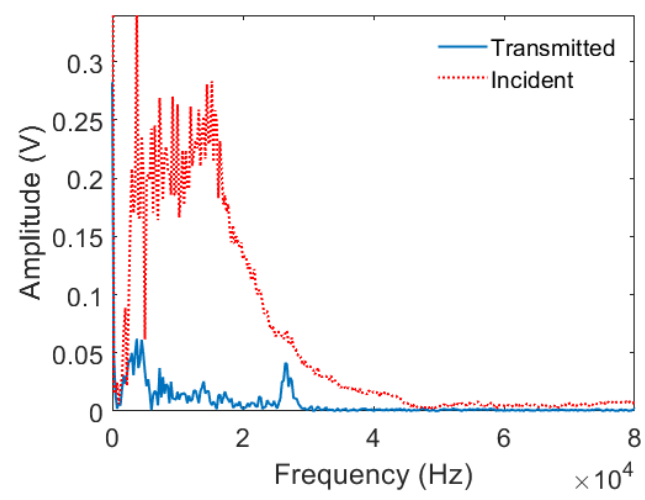

(b)

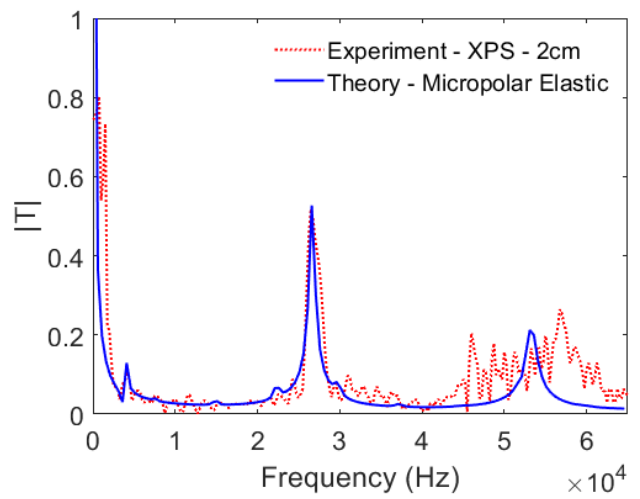

(c)

Figure 4: For the $2.0 \mathrm{~cm}$ thick XPS panel.(a) The temporal signals representing the incident and transmitted pressure (b) Their frequency spectrum. Comparisons between the theoretical and experimental, (c) the corresponding transmission coefficient.

${ }_{247} f_{c_{2} \mathrm{~cm}}=2171 \mathrm{~Hz}$ and $f_{c_{3 \mathrm{~cm}}}=3071 \mathrm{~Hz}$ ). The transmission coefficient dips at the cut-off

248 frequency (velocity of the transverse acoustic mode is $\infty$ ). The frequency of the first

249 peak representing the first mode in the EPS12 panel is lower than that of the XPS panel 
of the same thickness (Fig. 4 and Fig. 5F) meaning that the values of its micropolar 251 and elastic moduli are lower.

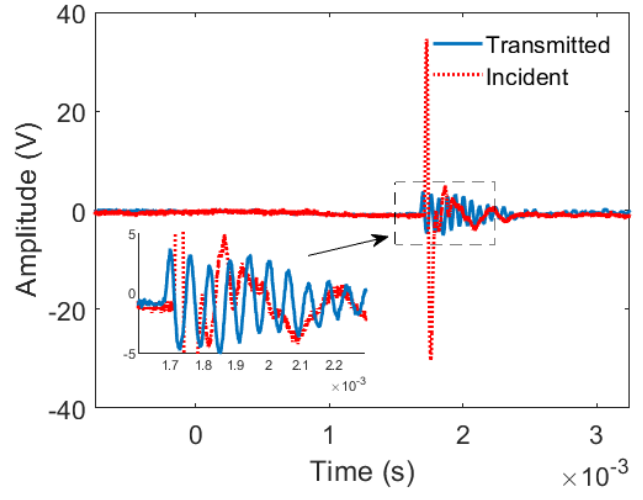

(a)

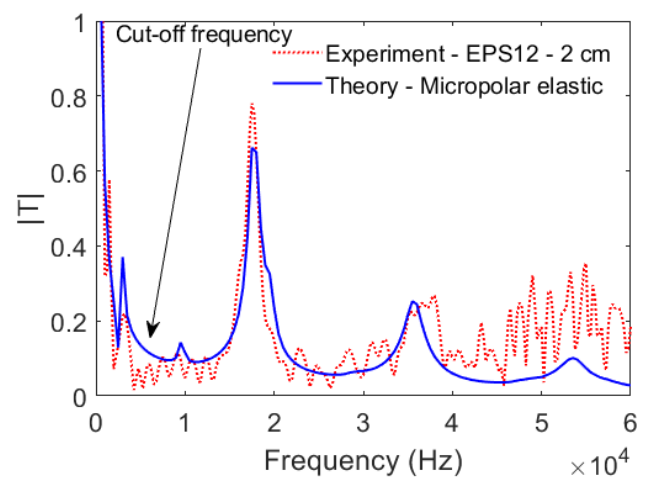

(c)

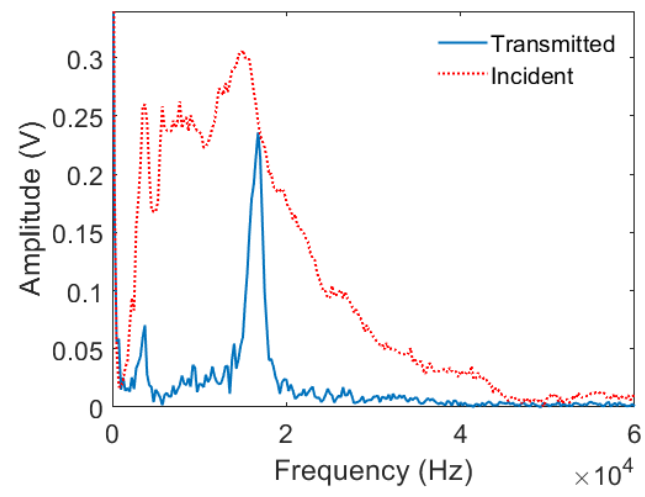

(b)

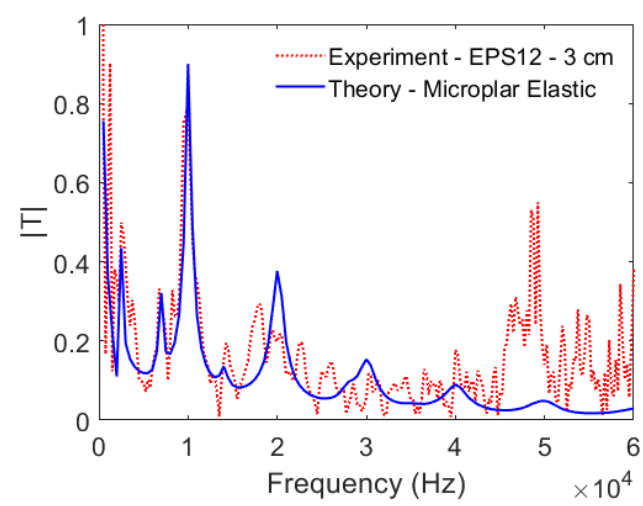

(d)

Figure 5: For the $2.0 \mathrm{~cm}$ thick EPS12 panel, (a) The temporal signals representing the incident and transmitted pressure (b) Their frequency spectrum. (c) Comparisons between the theoretical and experimental transmission coefficients. (d) The transmission coefficient for the $3.0 \mathrm{~cm}$ thick EPS12 panel

${ }^{252}$ The test results for an EPS12 $4.0 \mathrm{~cm}$ thick panel are shown in Fig. fig:A-4cm-thick ${ }_{253}$ xps panelc, (6). The acceleration (or early arrival) of the acoustic wave transmitted

${ }^{254}$ by the layer as compared to the incident acoustic pressure is more remarkable on the temporal signal of this panel, even without zooming. 


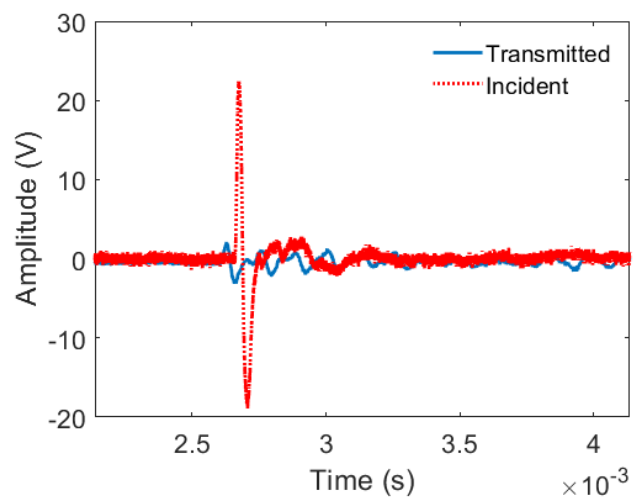

(a)

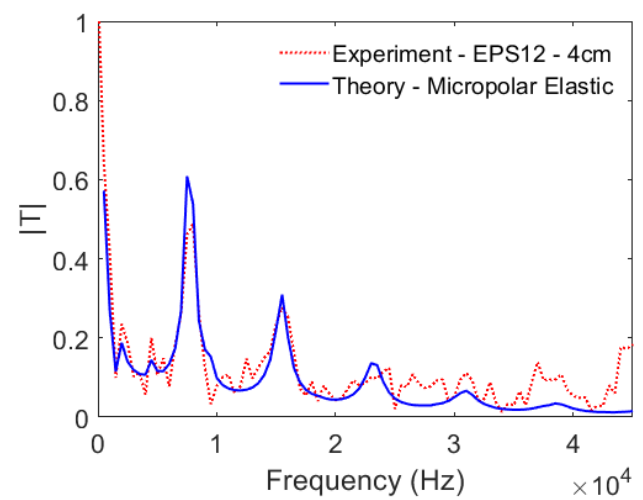

(c)

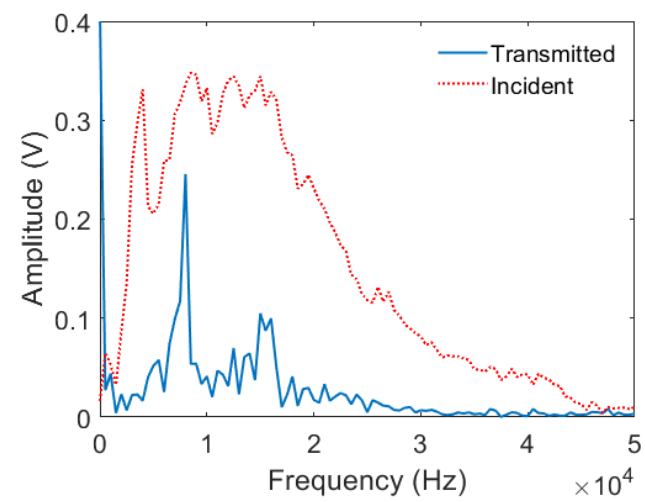

(b)

Figure 6: For the $4.0 \mathrm{~cm}$ thick EPS12 panel, (a) The temporal signals representing the incident and transmitted pressure (b) Their frequency spectrum. (c) Comparisons between the theoretical and experimental transmission coefficients.

Extended bandwidth using a $50 \mathrm{kHz}$ ultrasonic transducer as acoustic exciter source

The frequency band covered by the ribbon twitter loudspeaker was from $2-\approx 35 \mathrm{kHz}$

${ }_{258}$ (see Figs. 34, 4c, 5c and d, 66). The peaks of the modes were not well marked beyond

259 that bandwidth due to the low signal to noise ratio (SNR). This was due to the limited bandwidth of the loudspeaker. The SNR were lower for the thicker panels. In order to

261 cover the higher frequencies following i.e, from $\approx 30-90 \mathrm{kHz}$, an air coupled ultrasonic transducer was employed in place of the loudspeaker.

The incident temporal acoustic wave pressure signals and the comparison between the 
264 captured experimental and the computed theoretical transmission coefficients are shown

265 in Fig. (7). The spectra corresponding to the temporal signals are shown in Fig. (7)b.

266 The transmission coefficient is shown in Fig. (7)(c). The acoustic modes up to $120 \mathrm{kHz}$

267 are excited by this transducer and captured by the microphone. The transducer response

268 covers the high frequency zones where the loudspeaker response is lower, however, the

269 lower frequencies are not well reproduced with the former. The situation is improved

270 when the loudspeaker is employed because of its superior frequency response in the lower

271 frequency range.

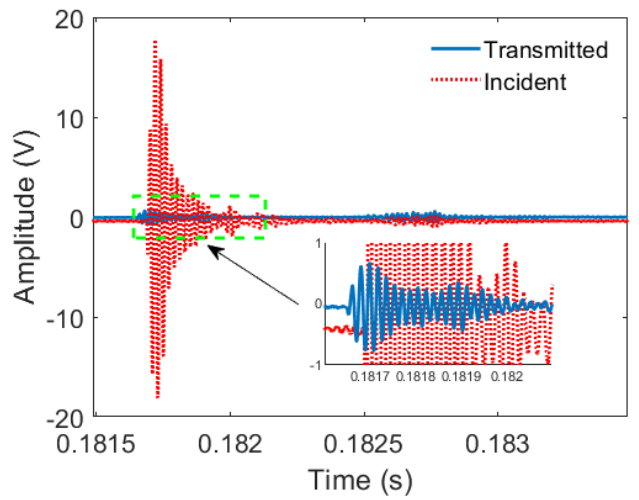

(a)

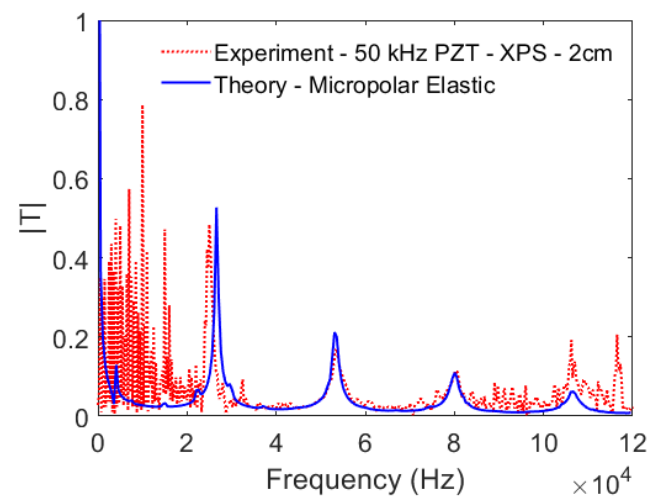

(c)

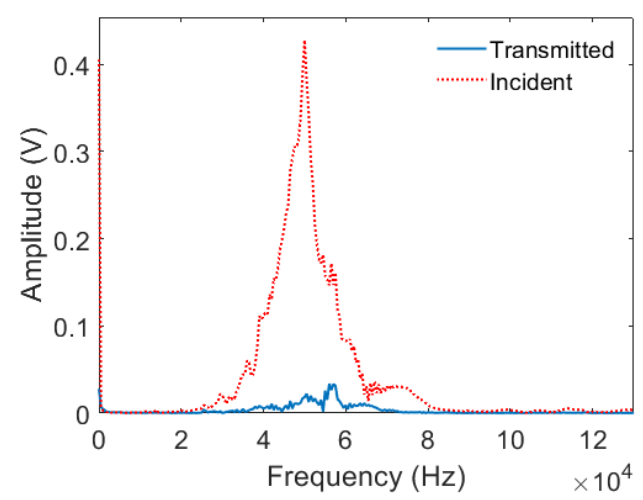

(b)

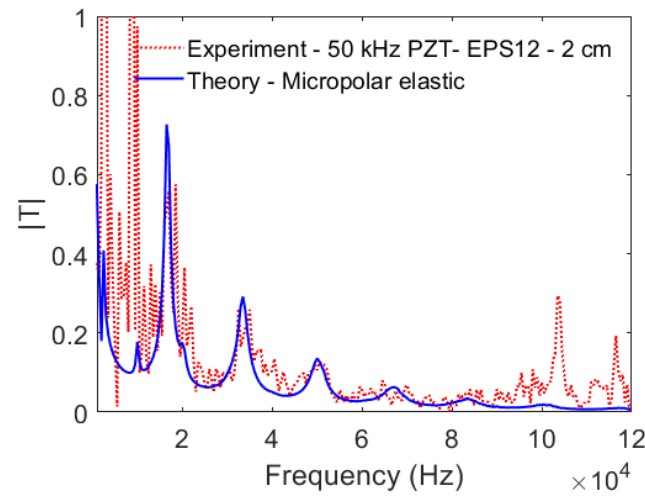

(d)

Figure 7: Using an Ultran air-coupled PZT transducer as acoustic source to probe a $2.0 \mathrm{~cm}$ XPS panel, (a) The incident and transmitted acoustic waves in the time domain, (b) Their spectrum. (c) Comparison between the theoretical and experimental transmission coefficients. (d) TC for the $2 \mathrm{~cm}$ thick EPS12 panel. 
Table 1: Adjusted parameters employed in the theoretical model to fit the experimental curves. The microinertia is $J$.

\begin{tabular}{|c|c|c|c|c|c|c|c|c|}
\hline Panel & $d_{1}(\mathrm{~cm})$ & $\rho\left(\mathrm{kg} / \mathrm{m}^{3}\right)$ & $E(\mathrm{MPa})$ & Loss $\chi$ & $\nu$ & $\mathcal{K}(\mathrm{MPa})$ & $\gamma(\mathrm{MN})$ & $J(N / m)$ \\
\hline XPS & 2 & 32 & 2.7 & 0.02 & 0.49 & 0.0510 & 1.68 & $2.35 \mathrm{e}-5$ \\
\hline XPS & 4 & 32 & 2.8 & 0.02 & 0.49 & 0.0510 & 0.57 & $2.35 \mathrm{e}-5$ \\
\hline EPS12 & 2 & 12 & 1.4 & 0.06 & 0.45 & 0.070 & 0.86 & $3.13 \mathrm{e}-5$ \\
\hline EPS12 & 3 & 12 & 1.5 & 0.06 & 0.43 & 0.070 & 1.2 & $3.13 \mathrm{e}-5$ \\
\hline EPS12 & 4 & 12 & 1.2 & 0.075 & 0.45 & 0.037 & 0.87 & $3.9 \mathrm{e}-5$ \\
\hline
\end{tabular}

\section{Discussion}

273 4.1. Comparison between the Young's modulus recovered with those obtained using other

$274 \quad$ methods

275 A summary of the micropolar parameters for the problem are tabulated in Table (1).

276 The precise optimized values will be given in a future study involving the resolution

277 of a complete inverse problem for wave propagation in Cosserat elastic continuum. A

${ }_{278}$ Manufacturer of EPS12 foams under the name of Geofoam (ACH Foam Technologiesß)

${ }_{279}$ (Denver, CO USA)), Geofoams (AFM Corporationß, Lakeville, MN USA) indicates that

280 the Elastic modulus measured using the ASTM D6817 standard [27, 28] at a compression

281 of $1 \%$ is $1500(\mathrm{kPa})$. Reference [37] also reports that the initial tangent modulus (deter-

282 mined from the linear portion of the stress-strain curve [37, 38] ) for EPS12 is $1600 \mathrm{kPa}$.

${ }_{283}$ These compare well with the value obtained from the experiment. The advantage of the

284 acoustic method is that it is non destructive and is lower compression rate as compared

285 to the Compression test methods where the materials are cut in cylindrical, rectangular

286 and cube form.

${ }_{287}$ 4.2. Phase wave velocities as a means of verification of some of the material parameters

${ }_{288}$ The phase wave velocity of the $\mathrm{P}$-wave modes (numbered $n$ ) propagating in the thick-

289 ness/layer of the panels are calculated from the following relationship

$$
v_{p}^{n} \approx 2 d_{1} \frac{f_{p}^{n}}{n}
$$


Table 2: Velocities calculated from the frequency of the first longitudinal mode $\left(f_{L_{1}}\right)$ and the recovered micropolar parameter $K$ and the P-wave modulus (longitudinal modulus) $M$. The cut-off frequency is

\begin{tabular}{cccccc}
$f_{c}$. & & & \\
Panel & density $\left(\mathrm{kg} / \mathrm{m}^{3}\right)$ & $d_{1}(\mathrm{~cm})$ & velocity $\left(\sqrt{\frac{M+\mathcal{K}}{\rho}}\right)(\mathrm{m} / \mathrm{s})$ & Velocity $\left(2 f_{L_{1}} d_{1}\right)(\mathrm{m} / \mathrm{s})$ & $f_{c}(\mathrm{~Hz})$ \\
\hline XPS & 32 & 4 & 1229 & 1240 & 6000 \\
XPS & 32 & 2 & 1066 & 1064 & 660 \\
EPS & 12 & 2 & 645 & 600 & 2171 \\
EPS & 12 & 3 & 620 & 640 & 3071 \\
EPS & 12 & 4 & 6242 & 600 \\
\hline \hline
\end{tabular}

where $d_{t h}$ is the panel thickness, $f_{p}^{n}$ is the frequency of the $n$th mode determined from the transmission coefficient. Velocity can also be computed once the theoretical TC data is fitted to the experimental TC and found satisfactory. The following relationships engaging the mechanical moduli and density $v_{p}^{1}=\sqrt{\frac{M+\mathcal{K}}{\rho}}$ from the theoretical model is employed.

Knowledge of the cut-toff frequency $f_{c}$ and the phase velocity are important for determining the bounds of $J$. The expression for the phase velocity is rewritten as $\left(v_{p}^{1}\right)^{2}=\left(M+2 \pi^{2} \rho J f_{c}^{2}\right)$. This shows that the velocity of the longitudinal modes also depend on the micropolar parameter $J$ (the micro-inertia) among others. The measured and recovered phase velocities are in good agreement (difference $<4$ percent) and are summarized in Table (2).

The influence of the Cosserat elastic constants and Poisson ratio on the transmission coefficient

The Sensitivity of the transmission coefficient vis-à-vis the micropolar parameters $\mathcal{K}$, $J, \gamma$ and the Poisson ratio was studied by varying them while all the others remained fixed at their expected values. Their influence was analyzed by examining the changes occurring on the magnitude of the transmission coefficient as parameters were varied. It was observed that $\mathcal{K}$ (the Cosserat couple modulus), influenced the magnitude of the transmission coefficient (Fig. (8)a and c) over the whole range of frequencies. The changes in magnitude of the TC with the variations of $\mathcal{K}$ were found to be well marked. This was accompanied by a directly proportional increase/decrease of the resonance 
311 frequency of the modes and as a consequence an advantage over the classical elastic model 312 that has only two tuning parameters $(E$ and $\nu)$. From this observation, the parameter $\mathcal{K}$ 313 can easily be recovered from experimental data since it has a significant influence on the 314 TC. The rotational inertia $J$ and the twist coefficient $\gamma$ influence the amplitudes around 315 the zoomed area of Figs. 8a and b. This is around the cut-off frequency area. Therefore 316 these two parameters can be considered as low frequency parameters. Poisson ratio was 317 also found to influence the TC (Figs. 8d)

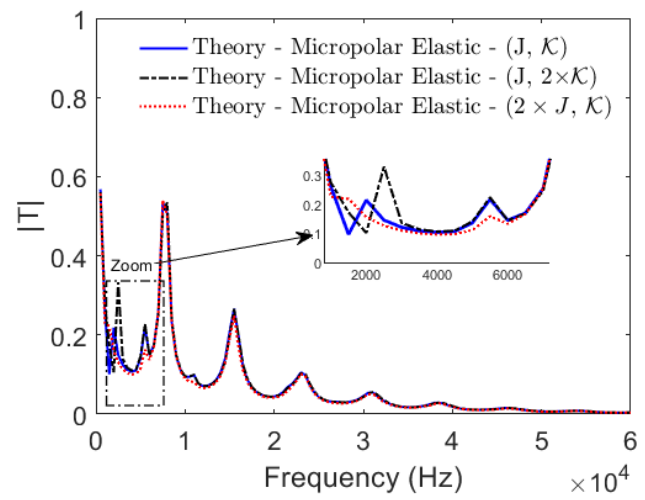

(a)

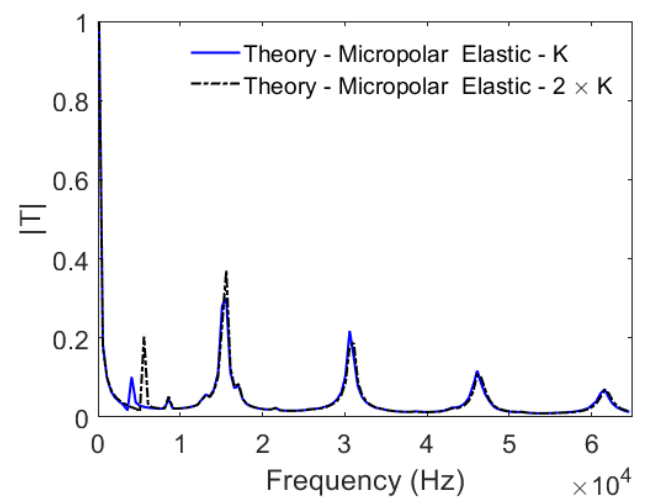

(c)

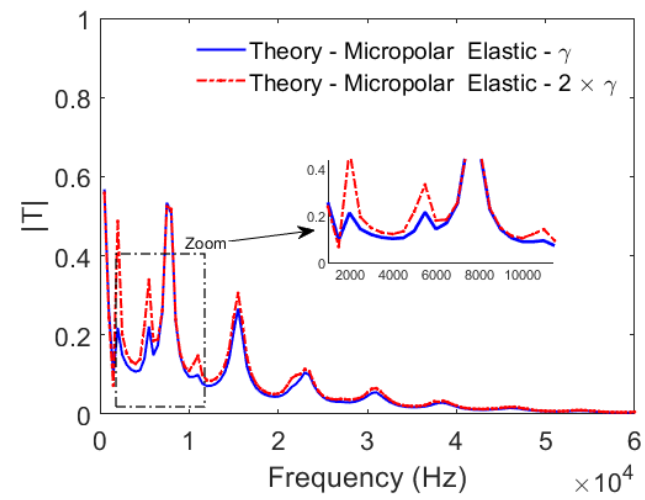

(b)

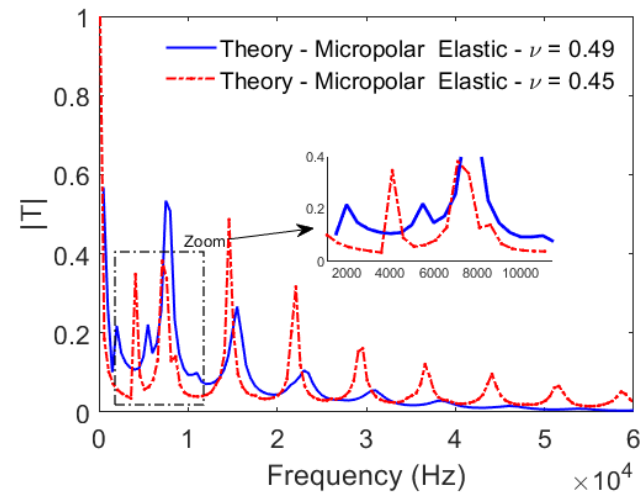

Figure 8: Sensitivity of the transmission coefficient to the variations of (a) The Cosserat couple modulus $\mathcal{K}(\mathcal{K}=42 \mathrm{kPa})$ and the rotational inertia $J=4.7 \times 10^{-5} \mathrm{~N} / \mathrm{m}$ and (b) The twist coefficient $\gamma=$ $5.6 \times 10^{5} \mathrm{~N}$ for an EPS12 $4.0 \mathrm{~cm}$ thick panel. For the XPS $4.0 \mathrm{~cm}$ thick panel (c) Variation of $\mathcal{K}$ $(\mathcal{K}=51 \mathrm{kPa})$. (d) Variation of Poisson ratio $\nu$. 
318 It is worth noting that the characteristic length [39, 40] can be obtained from the 319 micropolar twist $(\gamma)$ and couple $(\mathcal{K})$ modulus. This length is equal to the spherical 320 polystyrene bead diameter size that forms the EPS12 foam (their diameters are usually 321 in the range $0.3 \mathrm{~mm}-4 \mathrm{~mm})$. It is an intrinsic dimension defining the heterogeneous 322 structure of the material. The characteristic length in bending in micropolar theory was 323 given by Yang et al $\left[39\right.$, as $l_{b 2}=[\gamma /(2(2 \mu+\mathcal{K}))]^{1 / 2}$ in mm. In reference [41], $\mathcal{K}$ in $l_{b 2}$

324 was neglected. The beads from the $4 \mathrm{~cm}$ thick Eps12 foam had several diameters ranging

325 from $\approx 0.3 \mathrm{~mm}$ to $4 \mathrm{~mm}$. The calculations with the recovered values herein gave $l_{b 2} \approx 0.7$

$326 \mathrm{~mm}$.

327 4.3. Comparison between the theoretical micropolar elastic and classical elastic model

328 The micropolar model reproduces most of the small but different peaks (frequency 329 and amplitude) at the beginning of the transmission coefficient (around the cut-off fre3зо quency) and in the higher modes (Fig. 7). The transmission coefficients of the theoretical 331 micropolar elastic (modeling of an XPS $4 \mathrm{~cm}$ thick panel) and that of the classical elastic 332 continuum model and experiment were compared. The classical model was implemented s33 using the Transfer matrix method (TMM) in which the longitudinal and shear modes ${ }_{334}$ were taken into account [32]. Both formulations capture the resonance peaks in the 335 experimental TC curve (Fig. 9a). The absence of $J$ in the classical elastic model was 336 simulated to examine the effect of its absence on the TC of the micropolar elastic model.

337 It was given a very small value, $J=1 \times 10^{-50} \mathrm{Nm}^{-1}$ ( $J$ cannot be zero, it is denominator

${ }_{338}$ in the expressions for $\omega_{0}, c_{3}$ and $\left.c_{4}\right)$. The connection between the two theories is more 339 involved than merely making $J \rightarrow 0$ [14. The resulting TC had a peak around the 340 cut-off frequency missing (Fig. $9 \mathrm{~b}$ ). 


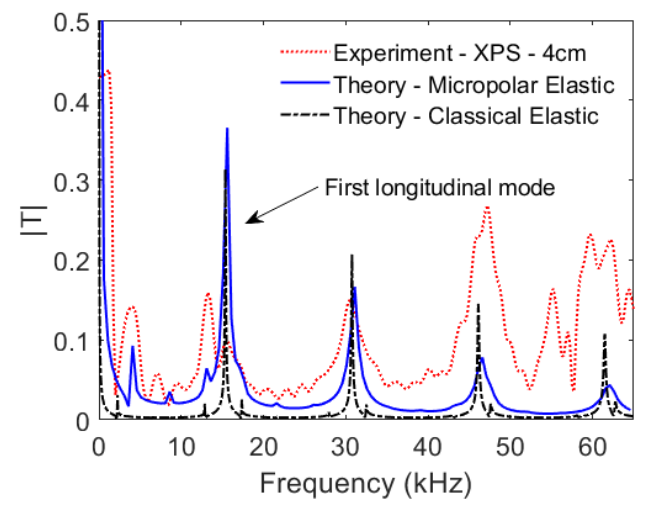

(a)

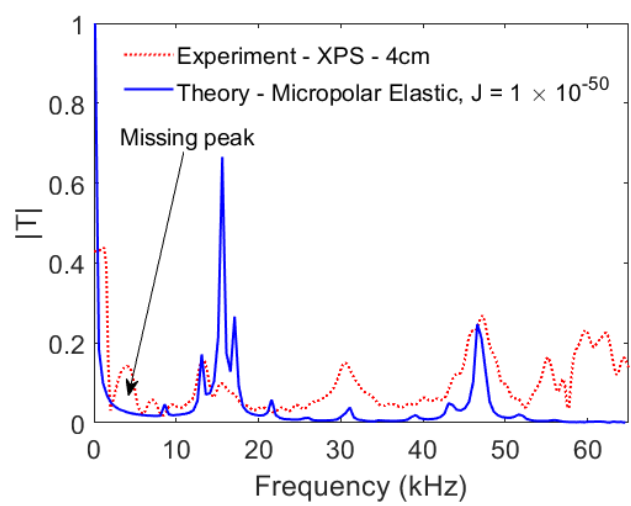

(b)

Figure 9: Comparison between the transmission coefficients for an XPS, $4.0 \mathrm{~cm}$ thick panel. (a) modeled by the micropolar elastic and the classical elastic formulations using the same $E$ and $\nu$. (b) The experimental and theoretical micropolar elastic model with a very small rotational inertia value, $J=1.0 \times 10^{-50} \mathrm{~N} / \mathrm{m}$.

\section{Conclusion}

An experimental test rig was setup for the obtention of the transmission coefficient in air to recover the micropolar elastic properties of XPS and EPS panels in the audible and ultrasonic frequency ranges. Not only did the transmission coefficients agree with the theoretical ones but the elastic parameters also agreed well with those in the literature for EPS12 of the same density but obtained using a different measurement technique.

It was confirmed for the materials tested (two families of closed-cell polystyrene foams, XPS and EPS) that the Cosserat continuum model, which admits additional degrees of freedom associated with rotation of the microstructure, described best their behavior. It is a better alternative to the classical continuum model. The absence of transmission at the cut-off frequency and a better correspondence of the transmitted modes with the experiment, were found to be the main features that differentiates the micropolar elastic material behavior from the classical elastic one. These features were more marked in the transmission coefficient curves for the thicker EPS and XPS foams.

The Cosserat couple modulus, $\mathcal{K}$, the micro-inertia $J$ and the twist modulus $\gamma$ were found to influence the transmission coefficient of XPS and EPS thick plates in air. This 
357 is an important finding because it implies that micropolar parameters: the micro-inertia

358 $J$ and $\mathcal{K}$ can be recovered using the measurement method developed herein. Recall that,

359 only $M$ and $\nu$ can be recovered using the classical elastic continuum theory [32].

360 Finally, this new method appeals to the characterization of closed-cell porous panels

361 and will provide elasticity data especially for the XPS foams that are hard to come by

362 in the available literature.

\section{Declaration of Competing Interest}

364 The authors declare that they have no known competing financial interests or personal 365 relationships that could have appeared to influence the work reported in this paper.

${ }_{366}$ Appendix A. The dispersion relationships

367 Plane wave type of propagation is assumed for which the potentials take the following 368 forms

$$
(q, \xi, \boldsymbol{\Pi}, \boldsymbol{\Theta})=(\bar{q}, \bar{\xi}, \overline{\boldsymbol{\Pi}}, \overline{\boldsymbol{\Theta}}) \exp [\mathrm{i}(\boldsymbol{k} \bullet \boldsymbol{r}-\boldsymbol{\omega} t)]
$$

369 where $\boldsymbol{k}$ is the wave-number vector $\left(\boldsymbol{k}=k_{x} \hat{x}+k_{y} \hat{y}+k_{z} \hat{z}\right), \boldsymbol{r}$ the position vector $(\boldsymbol{r}=$ 370 $x \hat{x}+y \hat{y}+z \hat{z}), \bar{q}, \bar{\xi}$ are complex amplitude constants, while $\overline{\boldsymbol{\Pi}}, \overline{\boldsymbol{\Theta}}$ are complex vectors.

${ }_{371}$ By using the vector identities in Appendix B and also writing $\nabla \times \boldsymbol{\Pi}=\mathrm{i} \boldsymbol{k} \times \boldsymbol{\Pi}$, 372 Eqn. (4) can be written

$$
\left(\omega^{2}-k^{2} c_{2}^{2}\right) \overline{\boldsymbol{\Pi}}+\mathrm{i} \frac{J \omega_{0}^{2}}{2} \boldsymbol{k} \times \overline{\boldsymbol{\Theta}}=0, \quad \mathrm{i} \frac{\omega_{0}^{2}}{2} \boldsymbol{k} \times \overline{\boldsymbol{\Pi}}+\left(\omega^{2}-\omega_{0}^{2}-c_{4}^{2} k^{2}\right) \overline{\boldsymbol{\Theta}}=0
$$

373 The dispersion relation is found through elimination of $\overline{\boldsymbol{\Pi}}$ and $\overline{\boldsymbol{\Theta}}$ from these equations

$$
\overline{\boldsymbol{\Theta}}=\frac{-i \omega_{0}^{2}}{2\left(\omega^{2}-\omega_{0}^{2}-c_{4}^{2} k^{2}\right)} \boldsymbol{k} \times \overline{\boldsymbol{\Pi}} \Rightarrow\left(\omega^{2}-k^{2} c_{2}^{2}\right) \overline{\boldsymbol{\Pi}}+\frac{J \omega_{0}^{4}}{4\left(\omega^{2}-\omega_{0}^{2}-c_{4}^{2} k^{2}\right)} \boldsymbol{k} \times \boldsymbol{k} \times \overline{\boldsymbol{\Pi}}=0
$$


374 Developing the expression $\boldsymbol{k} \times \boldsymbol{k} \times \overline{\boldsymbol{\Pi}}=(\boldsymbol{k} \bullet \overline{\boldsymbol{\Pi}}) \overline{\boldsymbol{\Pi}}-(\boldsymbol{k} \bullet \boldsymbol{k}) \overline{\boldsymbol{\Pi}}=-k^{2} \overline{\boldsymbol{\Pi}}$ (using the ${ }_{375}$ identity $\boldsymbol{A} \times(\mathbf{B} \times \boldsymbol{C})=(\boldsymbol{A} \bullet \boldsymbol{C}) \boldsymbol{B}-(\boldsymbol{A} \bullet \boldsymbol{B}) \boldsymbol{C}$ and $\left.\boldsymbol{k} \bullet \overline{\boldsymbol{\Pi}}=\boldsymbol{k} \bullet \overline{\boldsymbol{\Theta}}=0\right)$ then

$$
k^{4}+\left(\frac{\omega_{0}^{2}}{c_{4}^{2}}\left(1-\frac{J \omega_{0}^{2}}{4 c_{2}^{2}}\right)-\frac{\omega^{2}}{c_{2}^{2}}\left(1+\frac{c_{2}^{2}}{c_{4}^{2}}\right)\right) k^{2}+\frac{\omega^{2}}{c_{2}^{2} c_{4}^{2}}\left(\omega^{2}-\omega_{0}^{2}\right)=0
$$

376 which can also be written as a quartic equation of the type with solutions

$$
k^{4}-2 b k^{2}+c=0, \quad \Rightarrow \quad k_{3,4}^{2}=-b \pm \sqrt{b^{2}-4 c},
$$

377 where $b=\frac{\omega^{2}}{2 c_{2}{ }^{2}}\left(1+\frac{c_{2}{ }^{2}}{c_{4}{ }^{2}}\right)-\frac{\omega_{0}^{2}}{2 c_{4}{ }^{2}}\left(1-\frac{J, \omega_{0}^{2}}{4 c_{2}{ }^{2}}\right), c=\frac{\omega^{2}}{c_{2}{ }^{2} c_{4}{ }^{2}}\left(\omega^{2}-\omega_{0}^{2}\right)$

378 The two wave numbers, $k_{3}$ and $k_{4}$ are solutions and correspond to a transverse acoustic 379 mode (TA-mode) and transverse Optic mode (TO mode). The upper sign $(+)$ in the 380 equation of the coupled transverse displacement wave corresponds to $k_{3}$; the lower sign ${ }_{381}(-)$ corresponds to the wave number $k_{4}$ of the coupled transverse microrotational waves.

\section{Appendix B. Some vector calculus identities employed}

383 The following vector calculus identities were employed

$$
\begin{aligned}
& \nabla \bullet(\nabla \mathbf{q})=\nabla^{2} q, \nabla \bullet(\nabla \times \boldsymbol{\Pi})=0, \\
& \nabla \times(\nabla q)=0, \nabla \times(\nabla \times \boldsymbol{\Pi})=\nabla(\nabla \bullet \boldsymbol{\Pi})-\nabla^{2} \boldsymbol{\Pi}
\end{aligned}
$$

\section{References}

[1] E. Cosserat, F. Cosserat, Théorie des corps déformables, Librairie Scientifique, Hermann, Paris, 1909.

[2] W. Voigt, Theoretische studien fiber die elastizitatsverhiltnisse der kristalle (theoretical studies on the elasticity relationships of crystals), Abh. Gesch. Wissenschaften (1887) 34 .

[3] R. A. Toupin, Elastic materials with couple-stresses, Arch. Rational Mech. Ana. 11 (1962) $385-414$. [4] R. D. Mindlin, H. F. Tiersten, Effects of couple-stresses in linear elasticity, Arch. Rational Mech. Ana. 11 (1962) 415-488. 
[5] W. T. Koiter, Couple-stresses in the theory of elasticity, in: Pt. I-II. Proc. Koninkl., Neterland Akad., Wetensh B67, 1964, pp. 17-44.

[6] A. C. Eringen, Theory of micropolar elasticity, in: H. Liebowitz (Ed.), Fracture, Academic Press, New York, 1968, pp. 621-729.

[7] A. R. Hadjesfandiari, G. F. Dargush, Couple stress theory for solids, Int. J. Solids Struct. 48 (18) (2011) 2496-2510. URL https://www.sciencedirect.com/science/article/pii/S0020768311001727

[8] M. Brand, M. Rubin, A constrained theory of a cosserat point for the numerical solution of dynamic problems of non-linear elastic rods with rigid cross-sections, Int. J. Nonlin. Mech. 42 (2) (2007) 216232, special Issue in Honour of Dr Ronald S. Rivlin.

URL https://www.sciencedirect.com/science/article/pii/S0020746206000849

[9] A. C. Eringen, E. S. Suhubi, Elastodynamics, Vol. 2., Vol. Vol. 2, Academic Press, New York, 1975, Ch. 5 .

[10] A. C. Eringen, Linear theory of micropolar elasticity, J. Math. Mech. 15 (6) (1966) 909-923.

[11] C. Kafadar, A. Eringen, Micropolar media-i the classical theory, Int. J. Eng. Sci. 9 (3) (1971) 271 -305 .

URL http://www.sciencedirect.com/science/article/pii/0020722571900401

[12] R. Mindlin, Stress functions for a cosserat continuum, Int. J. Solids Struct. 1 (3) (1965) $265-271$. URL http://www .sciencedirect.com/science/article/pii/0020768365900338

[13] J. Altenbach, H. Altenbach, V. A. Eremeyev, On generalized Cosserat-type theories of plates and shells: a short review and bibliography, Arch. Appl. Mech. 80 (1) (2010) 73-92. URL https://doi.org/10.1007/s00419-009-0365-3

[14] S. Hassanpour, G. R. Heppler, Micropolar elasticity theory a survey of linear isotropic equations, representative notations, and experimental investigations, Math. Mech. Solids 22 (2) (2017) 224242 .

URL https : //doi.org/10.1177/1081286515581183

[15] S. Duan, W. Wen, D. Fang, A predictive micropolar continuum model for a novel three-dimensional chiral lattice with size effect and tension-twist coupling behavior, J. Mech. Phys. Solids 121 (2018) $23-46$. URL http://wWw.sciencedirect.com/science/article/pii/S0022509618303016

[16] A. Spadoni, M. Ruzzene, Elasto-static micropolar behavior of a chiral auxetic lattice, J. Mech. Phys. Solids 60 (1) (2012) $156-171$.

URL http://www.sciencedirect.com/science/article/pii/S0022509611001864

[17] R. S. Lakes, R. L. Benedict, Noncentrosymmetry in micropolar elasticity, Int. J. Eng. Sci. 20 (10) (1982) $1161-1167$. 
[18] R. Kumar, S. K. Tomar, Reflection and transmission of elastic waves at viscous liquid-micropolar elastic solid interface, Int. J. Math. Math. Sci. 26 (11) (2001) 685-694.

$430 \quad$ URL http://dx.doi.org/10.1155/S0161171201005415

[19] D. Singh, S. Tomar, Longitudinal waves at a micropolar fluid/solid interface, Int. J. Solids Struct. 45 (1) (2008) $225-244$. URL http: //wWW.sciencedirect.com/science/article/pii/S0020768307003071

[20] A. C. Eringen, Microcontinuum Field Theories - I. Foundations and Solids, 1st Edition, Classical and Continuum Physics, Springer-Verlag New York, New York, USA, 1999.

21] R. Lakes, W. J. Drugan, Bending of a cosserat elastic bar of square cross section: Theory and experiment, J. Appl. Mech. 82 (9) (2015) 091002-091010.

[22] H. Park, R. Lakes, Cosserat micromechanics of human bone: Strain redistribution by a hydration sensitive constituent, J. Biomech. 19 (5) (1986) $385-397$.

[23] R. Lakes, Experimental microelasticity of two porous solids, Int. J. Solids Struct. 22 (1) (1986) 55 $443-63$.

444 URL http://www.sciencedirect.com/science/article/pii/0020768386901034

445 [24] R. D. Gauthier, W. E. Jahsman, A quest for micropolar elastic constants, J. Appl. Mech. 42 (2) $446 \quad$ (1975) 369-374.

447 URL http://dx.doi.org/10.1115/1.3423583

[25] R. Lakes, Strongly Cosserat elastic lattice and foam materials for enhanced toughness, Cell. Polym. 12 (1993) $17-30$.

450 [26] W. B. Anderson, , R. S. Lakes, Size effects due to Cosserat elasticity and surface damage in closed451 cell polymethacrylimide foam, J. Mater. Sci. 29 (24) (1994) 6413-6419.

452 URL https://doi.org/10.1007/BF00353997

453 [27] ASTM C165-07(2012), Standard Test Method for Measuring Compressive Properties of Thermal In454 sulations, American Society for Testing and Materials (ASTM) International, West Conshohocken, $455 \quad$ PA (2012). [28] ASTM D6817 D6817M 15, Standard Specification for Rigid Cellular Polystyrene Geofoam, American Society for Testing and Materials (ASTM) International, West Conshohocken, PA (2015).

458 [29] V. R. Parfitt, A. C. Eringen, Reflection of plane waves from the flat boundary of a micropolar elastic half-space, J. Acoust. Soc. Am. 45 (5) (1969) 1258-1272.

URL https://doi.org/10.1121/1.1911598

461 [30] A. C. Eringen, Microcontinuum Field Theories: I. Foundations and Solids, Springer New York, 
463 [31] J. M. Carcione, Wave Fields in Real Media, 1st Edition, Vol. 31 of Handbook of Geophysical Exploration Seismic Exploration, Pergamon, Amsterdam, 2001.

32] E. Ogam, Z. Fellah, G. Ogam, Identification of the mechanical moduli of closed-cell porous foams using transmitted acoustic waves in air and the transfer matrix method, Compos. Struct. 135 (2016) $205-216$

468 [33] L. J. Gibson, M. F. Ashby, Cellular solids : Structure and properties, 2nd Edition, Cambridge Solid State Science, Cambridge University Press, 1997.

470 [34] I. MathWorks, Signal Processing Toolbox for Use with MATLAB: User's Guide, version 7.2 (r2016a) Edition, Computation, visualization, programming, The MathWorks, Natick, MA USA, 2016.

472 [35] A. Nuttall, Some windows with very good sidelobe behavior, IEEE Trans. Acoust., Speech, Signal Process. 29 (1) (1981) 84-91.

474 [36] E. Ogam, Z. Fellah, N. Sebaa, J.-P. Groby, Non-ambiguous recovery of Biot poroelastic parameters of cellular panels using ultrasonic waves, J. Sound Vib. 330 (6) (2011) 1074 - 1090. URL http://www.sciencedirect.com/science/article/pii/S0022460X10006371

[37] Y. Beju, J. Mandal, Expanded polystyrene (eps) geofoam: Preliminary characteristic evaluation, Procedia Eng. 189 (2017) 239 - 246, proceedings of the International Scientific Conference Transportation Geotechnics and Geoecology (TGG-2017). URL http://wWw.sciencedirect.com/science/article/pii/S1877705817321598

[38] ASTM D1621 - 10, Standard Test Method for Compressive Properties Of Rigid Cellular Plastics, American Society for Testing and Materials (ASTM) International, West Conshohocken, PA (2010). [39] J. Yang, R. S. Lakes, Experimental study of micropolar and couple stress elasticity in compact bone in bending, Journal of Biomechanics 15 (2) (1982) 91-98. URL https://www.sciencedirect.com/science/article/pii/0021929082900409 [40] F.-Y. Huang, B.-H. Yan, J.-L. Yan, D.-U. Yang, Bending analysis of micropolar elastic beam using a 3-D finite element method, International Journal of Engineering Science 38 (3) (2000) 275-286. URL https://www.sciencedirect.com/science/article/pii/S0020722599000415

[41] Z. Rueger, R. S. Lakes, Cosserat elasticity of negative poisson's ratio foam: experiment, Smart Materials and Structures 25 (5) (2016) 054004. 